\title{
Profiling Analysis of Fatty Acids and Collagens Obtained from Sea Cucumbers
}

\author{
Pascal Budzinski ${ }^{1}$, Mananya Maimeun ${ }^{2}$, Parita Mutrakulcharoen ${ }^{2,3}$, Benjamaporn Wonganu ${ }^{4}$, and Malinee Sriariyanun ${ }^{2, *}$
}

\begin{abstract}
${ }^{1}$ Institute of Thermodynamics, Helmut-Schmidt-University / University of the Federal Armed Forces Hamburg, Hamburg, Germany ${ }^{2}$ Department of Mechanical and Process Engineering, The Sirindhorn International Thai-German Graduate School of Engineering (TGGS), King Mongkut's University of Technology North Bangkok (KMUTNB), Bangkok, Thailand

${ }^{3}$ Department of Agro-Industrial, Food, and Environmental Technology, Faculty of Applied Science, King Mongkut's University of Technology North Bangkok (KMUTNB), Bangkok, Thailand
\end{abstract}

${ }^{4}$ Department of Biotechnology, Faculty of Applied Science, King Mongkut's University of Technology North Bangkok (KMUTNB), Bangkok, Thailand

\begin{abstract}
Investigations of alternative resources for production of functional foods and ingredients containing valuable compounds with biological activities are getting more and more attention. Sea cucumbers are aquatic functional foods with various medical and pharmaceutical effects, such as antioxidant, antibacterial, antifungal, antiviral, anti-inflammatory and neuroprotective activities. This study aimed to conduct profiling analysis of fatty acids and collagens extracted from four different sea cucumbers harvested from Papua New Guinea by using Gas Chromatrography-Mass Spectrometry (GC-MS) and Fourier Transform Infrared Spectrometer (FT-IR). Three different extraction methods in combination with various solvents were used to find the best combination for extracting fatty acids. Enzymatic and chemical extraction methods were applied for collagen extraction. The highest fat recovery in this study was $85.32 \%$ of theoretical yield with high proportions of unsaturated fatty acids up to $74.54 \%$, and enriched with omega3 fatty acid. FT-IR chromatogram of sea cucumber protein extracts showed the characteristic of collagen enriched with glycine and proline. The nutritional analysis of sea cucumber extracts demonstrated the potential use as functional foods and ingredients with high benefits to human health.
\end{abstract}

Keyword. Sea cucumber, Fatty acid profiling, Collagen, Omega 3, Functional food

\section{Introduction}

Due to the growing needs of functional foods and ingredients in the markets worldwide, finding the raw materials with beneficially nutritional properties and specific to targeted consumers gains intensive attention from industries. For example, the food supplements with human health improvement effects obtained from animal meats, i.e. nuts and herbs, may contain active chemicals, which stimulate allergenic reactions to some sensitive consumers [1]. Therefore, variations of raw material sources are important for production and development of foods and ingredients to supply the industries and markets.

Among marine organisms, sea cucumbers are interesting feedstocks with pharmaceutical activities. With these characteristics, they could be used in foods as well as biomedicine industries [2]. Recently, many studies provide scientific evidences that sea cucumbers contain various biologically active compounds that provide health benefits, such as antioxidant, antibacterial, antifungal, antiviral, anti-inflammatory activities and neuroprotective activities [3].
Proximate analysis revealed that wet sea cucumber consists of $44-55 \%$ protein, $3-5 \%$ carbohydrate and $1.5 \%$ fat suggesting that it is low-fat, high protein food ingredients [4]. Collagen is one of the major protein found in animal connective tissues. It has been described to function in various effects, including damaged tissue repairing [5], antitumor [6], antioxidant [7], and angiotensin-converting enzyme inhibitory activity [8]. Therefore, collagen is one of major active components in cosmetic recipes. Although, sea cucumber contains low quantity of fat, the fatty acid profiles are rich with high health-value components, such as eicosatetraenoic acid (EPA) and docosahexaenoic acid (DHA) [4]. EPA and DHA have attracted much attention for the beneficial effects on human health in reducing the risk for sudden death caused by cardiac arrhythmias and heart diseases [9, 10]. Additionally, they could be used as a wound healing agent or an antithrombotic [10]. As the consumption of DHA and EPA obtained from foods contributes only a very small proportion to total daily need, the use of dietary supplements containing EPA and DHA is required for total daily intakes.

However, the variations in supplied sea cucumbers in terms of species, quantity and quality cannot secure their

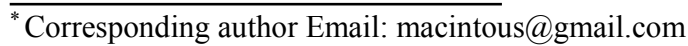


functions as raw materials in industries. Additionally, other physical factors, including seasons, harvesting areas and post-harvest manipulations also affect the active compounds in sea cucumbers [11]. Therefore, the objectives of this work targeted to the analysis of the fatty acid profiles and collagen obtained from 4 sea cucumbers harvested in Papua New Guinea. The investigation results here could contribute in the selection of sea cucumbers to supply the market needs.

\section{Material and methods}

\section{1 sample preparation}

Samples of dried Blackfish (Holothuria nobilis), Black Teatfish (Actinopyga miliaris), White Teatfish (Holothuria fuscogilva), and Elephant Trunkfish (Holothuria fuscopunctata) were delivered from Papua New Guinea to the laboratory (Courtesy provided by Wonnapob Co., Ltd., Bangkok, Thailand). The dry weight and the wet weight were measured and recorded. For the wet weight, the sea cucumbers were rehydrated, by putting them into deionized water for 4 days at $4{ }^{\circ} \mathrm{C}$. After recording the weights, the sea cucumbers were sliced into small pieces and stored at $-18^{\circ} \mathrm{C}$.

\subsection{Fatty acid extraction}

In order to find the best method for fat extraction from sea cucumber, three different extraction methods, including Soxhlet extraction [12], Reflux and Bligh and Dyer [13], were comparatively tested with the same solvent, a mixture of chloroform and methanol in a 1:1-ratio (v/v). The ratio of sample:solvent was fixed at $10 \% \mathrm{w} / \mathrm{v}$. The setting for the different extraction methods was shown in Table 1. Each extraction method was performed twice on each sea cucumber.

Table 1. Settings for the different extraction methods.

\begin{tabular}{lccc}
\hline \multicolumn{1}{c}{ Parameters } & Soxhlet & Reflux & Bligh\&Dyer \\
\hline Wet weight $(\mathrm{g})$ & 20 & 10 & 10 \\
Chloroform $(\mathrm{mL})$ & 100 & 50 & 50 \\
Methanol $(\mathrm{mL})$ & 100 & 50 & 50 \\
Temperature $\left({ }^{\circ} \mathrm{C}\right)$ & 84 & 75 & 30 \\
\hline
\end{tabular}

For Bligh and Dyer extraction [13], the sample was transferred to a tube. The sample and the $3 \mathrm{~mL}$ of solvent were homogenized for $20 \mathrm{~min}$ with a homogenizer (D-130, Wiggens, Straubenhardt, Germany). After homogenization, $1.8 \mathrm{~mL}$ water for each gram of sample was added. The tube was shaken for $2 \mathrm{~h}$ before the mixture was transferred to a centrifugation tube. After centrifugation for $10 \mathrm{~min}$ at $4,000 \mathrm{~g}$, the top layer (aqueous phase) was discarded, the lower layer (organic phase) was collected. After solvent evaporation, the fat remains in the tube and can be analyzed by GC-MS. In case of Soxhlet and Reflux extraction, the steps were conducted as described previously [14]. The sample was transferred into a timble in the glass apparatus. After adding the solvent, both extractions were performed for $6 \mathrm{~h}$ in the waterbath at the temperature from Table 1. Then, the solvent was filtrated with filter paper before it was evaporated, and fat sample was collected for GC-MS analysis.

After finding a suitable extraction method, the selected method was modified with different solvent mixtures, including acetic acid, hexane, chloroform, methanol and ethanol. These solvents were selected to cover a wide variety in the polarity and property of organic solvents.

\subsection{Fatty acid analysis}

For analysing the profiles with the GC-MS, the samples were derivatized [15], using $2 \mathrm{~mL}$ of $1 \% \mathrm{H}_{2} \mathrm{SO}_{4}$ in Methanol (MtOH) for each $40 \mu \mathrm{g}$ of fat. This mixture was boiled at $70^{\circ} \mathrm{C}$ for $2 \mathrm{~h}$ and every $30 \mathrm{~min}$ the sample was vortexed. After $2 \mathrm{~h}$, the sample was cooled to room temperature. $3 \mathrm{~mL}$ of hexane and $2 \mathrm{~mL}$ of deionized water were added to separate the fatty acids from aqueous phase. After centrifugation at 4,000 g for $10 \mathrm{~min}$, the top layer was collected and evaporated to weigh the recovered amounts of fatty acids. For GC-MS the dry fatty acids were dissolved in hexane to a concentration of $50 \mu \mathrm{g} / \mu \mathrm{l}$ and filtered with a $0.2 \mu \mathrm{m}$ filter. The profiles of the extracted fat were analysed with a GC-MS (QP2020, Shimadzu, Japan) using a DB-WAX capillary column (Agilent Technologies, Inc., CA., USA)(30 m x $0.25 \mathrm{~mm}, 0.25 \mu \mathrm{m})$ [16]. The carrier gar was helium with flow rate of 1.0 $\mathrm{mL} / \mathrm{min}$. The oven temperature programmed was initially set at $120^{\circ} \mathrm{C}$ and increased to $220^{\circ} \mathrm{C}$ at the rate of $10^{\circ} \mathrm{C} / \mathrm{min}$. Then, the temperature increased to $250^{\circ} \mathrm{C}$ with the rate of $15^{\circ} \mathrm{C} / \mathrm{min}$. The $1 \mu \mathrm{l}$ of sample was injected using a split mode with the split ratio of $1: 30$. The mass spectrometer was set to scan in range of $\mathrm{m} / \mathrm{Z} 35-550$ in electron ionization mode (EI) at $70 \mathrm{eV}$.

\subsection{Collagen extraction}

In this study, enzymatic and chemical extraction methods were used to recover collagen from sea cucumbers. All these procedures were carried out at $4^{\circ} \mathrm{C}$ in order to reduce the enzymatic activities. In case of the enzymatic pepsin digestion [17], $200 \mathrm{~mL}$ of $0.5 \mathrm{M}$ acetic acid were added to $20 \mathrm{~g}$ of sea cucumber sample, and homogenized for 20 min. After homogenization, pepsin (Sigma-Aldrich, Inc., USA) was added with a ratio of 1:100 v/w, and incubated at $4{ }^{\circ} \mathrm{C}$ for $16 \mathrm{~h}$. To collect the supernatant, the mixture was centrifuged at $4,000 \mathrm{~g}$ for $40 \mathrm{~min}$. Collagen was extracted from the supernatant by salt precipitation, $2.6 \mathrm{M}$ $\mathrm{NaCl}$ were added and stirred overnight. After centrifugation at 4,000 $\mathrm{g}$ for $40 \mathrm{~min}$, the precipitate was collected and dialyzed against deionized water for $24 \mathrm{~h}$. The remaining extract was called pepsin-solubilized collagen (PSC).

Chemical cleavage can be achieved by treatment with diluted solutions of formic acid, hydrochloric acid or acetic acid [18]. In this work, $20 \mathrm{~g}$ of sample were stirred in $500 \mathrm{~mL}$ of distilled water for $30 \mathrm{~min}$, the water was replaced again to clean up the sample. After $1 \mathrm{~h}$ stirring, the water was discarded and $500 \mathrm{~mL}$ of the disaggregation solution (containing $4 \mathrm{mM}$ ethylene-diaminetetraacetic acid (EDTA), 0.1 M Tris-HCl, pH 8.0) was added and 
stirred for 3 days. The liquid was replaced with $500 \mathrm{~mL}$ of distilled water and stirred for 2 days. Then, the supernatant was collected by centrifugation at 4,000 $\mathrm{g}$ for $10 \mathrm{~min}$. Then, crude collagen fibrils were collected from supernatant fraction by centrifugation at $4,000 \mathrm{~g}$ for 40 $\min$.

\subsection{Collagen analysis}

The extracted collagen was analysed by SDS-PAGE by mixing with SDS-PAGE loading buffer. The mixture was denatured by heating at $95^{\circ} \mathrm{C}$ for $10 \mathrm{~min}$. The denatured proteins were separated under electrophoresis and stained with Coomassie blue dye [19].

For FT-IR analysis, the extracted collagen fibrils were air dried at $60^{\circ} \mathrm{C}$ overnight and then spectrum scans were obtained from 4000 to $650 \mathrm{~cm}^{-1}$. FT-IR spectra were carried out to compare the molecular groups of the extract with a standard collagen FT-IR profile.

\section{Results and discussion}

\subsection{Selection of fatty acid extraction method}

In order to select the most efficient extraction method for fat, the recovered yields and fatty acid profiles obtained from 3 different methods, including Soxhlet extraction, Reflux and Bligh and Dyer, were compared by using the Blackfish and Black Teatfish as models. Based on the recovered fat yields, higher fat contents could be obtained from Blackfish (ranging from 1.25-2.06 $\mathrm{wt} \%$ of dried weight) compared to Black Teatfish (ranging from 0.18$0.49 \mathrm{wt} \%$ of dried weight) by using all 3 extraction methods (Table 2). In both sea cucumbers, Bligh and Dyer method provided the highest recovered fat yields compared to the other methods.

In addition to fat yield, the fatty acid profiles obtained from 3 different extraction methods were also analysed by using GC-MS analysis. The fatty acids were classified to 2 main groups, saturated fatty acid (SFA) and unsaturated fatty acid (USFA). In Black Teatfish, the SFA and USFA contents were in similar level at $46.42 \%$ and $45.00 \%$, respectively (Table 2). While in Blackfish, USFA was $53.1 \%$ and SFA was $43.1 \%$, suggesting that this sea cucumber contained high content of USFA. Using 3 different extraction methods, the proportions of USFA: SFA in both sea cucumbers were similar, suggesting that the profiles of fatty acids did not much affected by extraction methods.

Among USFA, there are subgroups of fatty acids called omega fatty acids, i.e. omega $3(\omega-3)$. To be considered as good fatty acids with benefits to human health, thus, the high $\omega^{-3}$ fat content should be included in the daily meal $[1,9]$. In both sea cucumbers, nearly half of the USFA are out of $\omega-3$ and $\omega-6$ fatty acids (Table 2 ). Also, the ratios between $\omega-3$ and $\omega-6$ fatty acids in both sea cucumbers were considered to be the recommended fats. It is estimated that the $\omega-6$ to $\omega-3$ ratio in the modern diet is 20:1 when it should ideally be closer to 2:1 [9]. This nutrition imbalance can lead the health problems including heart disease, obesity, diabetes and even cancer [20]. The ratios of the extracted $\omega-6$ to $\omega-3$ fatty acids from Black Teatfish and Blackfish were $1: 2$ to $1: 3$ (Table 2), which were close to the ideal $\omega-6$ to $\omega-3$ ratio.

Table 2. Recovered fat yields and fatty acid profiles of Black Teatfish and Blackfish obtained from three extraction methods.

\begin{tabular}{lccc}
\hline $\begin{array}{c}\text { Type of fatty } \\
\text { acid }\end{array}$ & Bligh\&Dyer & Reflux & Soxhlet \\
\hline Black Teatfish & & & \\
Yield (wt $\%$ & $0.49 \pm 0.05$ & $0.38 \pm 0.14$ & $0.18 \pm 0.06$ \\
dried weight) & $46.42 \pm 0.29$ & $42.04 \pm 0.88$ & $36.71 \pm 1.49$ \\
$\Sigma$ SFA & $45.00 \pm 1.38$ & $48.92 \pm 1.41$ & $49.87 \pm 1.95$ \\
$\Sigma$ USFA & $7.14 \pm 0.23$ & $6.93 \pm 0.42$ & $8.72 \pm 1.45$ \\
$\quad \Sigma(\omega-3)$ & $19.14 \pm 0.58$ & $18.20 \pm 1.36$ & $19.13 \pm 0.54$ \\
$\Sigma(\omega-6)$ & $5.57 \pm 0.45$ & $5.96 \pm 1$ & $5.56 \pm 1.49$ \\
$\quad \Sigma(\omega-9)$ & & & \\
\hline Blackfish & & & \\
Yield $(w t \%$ & $2.06 \pm 0.26$ & $1.24 \pm 0.09$ & $0.15 \pm 0.08$ \\
dried weight) & & & \\
$\Sigma$ SFA & $43.1 \pm 0.78$ & $43.92 \pm 0.93$ & $43.25 \pm 2.12$ \\
$\Sigma$ USFA & $53.1 \pm 0.64$ & $54.35 \pm 0.94$ & $55.62 \pm 0.92$ \\
$\Sigma(\omega-3)$ & $6.98 \pm 0.39$ & $7.62 \pm 0.5$ & $7.25 \pm 0.85$ \\
$\Sigma(\omega-6)$ & $13.55 \pm 0.5$ & $15.80 \pm 1.62$ & $15.97 \pm 1.45$ \\
$\Sigma(\omega-9)$ & $3.67 \pm 1.18$ & $3.96 \pm 2.27$ & $5.72 \pm 3.5$ \\
\hline
\end{tabular}

The highest amount of essential fatty acids has the eicosapentaenoic acid (EPA, C20:5, $\omega^{-3}$ ) and arachidonic acid (ARA, C20:4, $\omega-6$ ). The amount of EPA in the Black Teatfish was 5-6.5 wt \% and the amount of ARA was 11$14 \mathrm{wt} \%$ of the fat. For the Blackfish, the amount of EPA was 5-5.5 wt $\%$ and the amount of ARA was 11-14 wt\% of the fat. EPA and ARA were polyunsaturated fatty acids (PUFA) with important biological functions in human health as they are components of phospholipids of cell membranes, precursors in neurotransmitter, and signaling molecules. They are abundantly found in brain, muscle and livers, and, with docosahexaenoic acid (DHA), ARA contributed to $\sim 20 \mathrm{wt} \%$ of fatty acids in human brain [9].

The effect of different extraction methods was evaluated by comparing each group of beneficial fatty acids. The results show that the extracted profiles for each method were similar with small differences (Table 2). Regarding to yields and profiles, Bligh and Dyer method was the best of the three methods. In addition, it was the most time efficient method among those three methods. The Bligh and Dyer method had only an extraction time of $2 \mathrm{~h}$, while the other methods had an extraction time of $6 \mathrm{~h}$. Therefore, the later part of this study, Bligh and Dyer method was used for further fat analysis.

\subsection{Selection of fatty acid extraction solvent}

In 3.1, methanol:chloroform (MtOH:Chlo) solvent was selected to be the tested solvent as suggested by other studies. In this study, different solvent systems, including hexane (Hex), toluene (Tol), methanol $(\mathrm{MtOH})$, ethanol $(\mathrm{EtOH})$, acetic acid (Acet) and chloroform (Chlo), were challenged in fat extraction using the Bligh and Dyer 
method. In this experiment, White Teatfish was used as a model sample for solvent type testing.

For the single solvent testing, including Hex, Tol and $\mathrm{MtOH}$, the best tested solvent was the protic solvent $\mathrm{MtOH}$ with an extracted yield of $0.21 \mathrm{wt} \%$, but it still could not reach the original mixture of $\mathrm{MtOH}$ :Chlo with an extracted amount of $0.49 \mathrm{wt} \%$ (Fig. 1). The first assumption for the $\mathrm{MtOH}$ :Chlo mixture extracting the highest yield could be due to the strength of the acid-base reaction. The reaction takes place due to the strength difference of the acid and base given by the $\mathrm{pK}_{\mathrm{a}}$. value. The $\mathrm{pK}_{\mathrm{a}}$ value difference between Chlo $\left(\mathrm{pK}_{\mathrm{a}}=25\right)$ and $\mathrm{MtOH}\left(\mathrm{pK}_{\mathrm{a}}=15.5\right)$ is 9.5. With pairing the mixture of acetic acid $\left(\mathrm{pK}_{\mathrm{a}}=4.76\right)$ and hexane $\left(\mathrm{pK}_{\mathrm{a}}=51\right)$ as solvent, the $\mathrm{pK}_{\mathrm{a}}$ difference is 46.24 , which is much higher than MtOH:Chlo mixture [21]. The second assumption could be the amounts of available $\mathrm{OH}$ groups to perform a proton transfer. In this case, the $\mathrm{MtOH}$ is the shortest chained alcohol and offers the highest amounts of $\mathrm{OH}$ groups.

However, the assumption of $\mathrm{pK}_{\mathrm{a}}$ difference did not really support the results of other mixed solvents. The extracted fat yield with Acet and Hex was even lower with bigger $\mathrm{pK}_{\mathrm{a}}$ difference (Fig. 1). On the other hands, comparing between numbers of reactive groups available in $\mathrm{MtOH}$ and Acet in the same solvent volume, $\mathrm{MtOH}$ has more numbers than Acet because $\mathrm{MtOH}$ has less molar mass $\left(\mathrm{M}_{\mathrm{CH} 3 \mathrm{OH}}=32.04 \mathrm{~g} / \mathrm{mol}, \mathrm{M}_{\mathrm{CH} 3 \mathrm{COOH}}=60.05 \mathrm{~g} / \mathrm{mol}\right)$. Based on the second assumption, the mixture of EtOH and Chlo should extracted a lower amount than the mixture $\mathrm{MtOH}$ and Chlo, because the EtOH mix provides less numbers of $\mathrm{OH}$ groups in the same solvent volume $\left(\mathrm{M}_{\mathrm{MtOH}}=32.04\right.$ $\mathrm{g} / \mathrm{mol}, \mathrm{M}_{\mathrm{EtOH}}=46.07 \mathrm{~g} / \mathrm{mol}$ ). Interestingly, the fat yield before fat derivatization obtained from $\mathrm{MtOH}$ and Acet mix (0.96 wt \%) was higher than EtOH and Acet mix (0.68 wt $\%$ ) that supported the possibility of the second assumption.

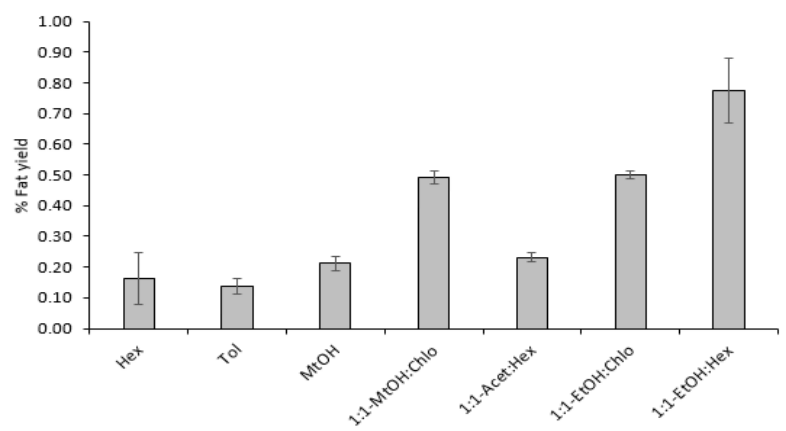

Fig. 1. Fat extraction from White Teatfish by using different solvent system.

Then, the pairing mix of EtOH was changed from Chlo to Hex and the highest fat yield was up to $0.78 \mathrm{wt} \%$. To confirm the selection of extraction solvents, the MtOH:Chlo, EtOH:Chlo and EtOH:Hex were repeated in the Elephant Trunkfish. The fat yields obtained from three different mixed solvents show comparable level at 0.56$0.59 \mathrm{wt} \%$ (Table 3). For fatty acid profiles, EtOH:Hex extracted USFA from White Teatfish up to $74.54 \mathrm{wt} \%$, which was higher than that obtained from $\mathrm{MtOH}$ :Chlo and
EtOH:Chlo. While, in Elephant Trunkfish, three different mixed solvents provided the similar level of USFA. The compositions of the USFAs showed clearly that the EtOH:Hex provided not only the highest yield of USFA, it also extracted the highest amounts of $\omega^{-3}(11.13 \mathrm{wt} \%)$ and $\omega-6$ fatty acids (37.19 wt\%) (Table 3$)$. This extracted fat had a healthy $\omega-3$ to $\omega-6$ fatty acid ratio of about $1: 3$, a good ratio of healthy fat [20].

Table 3. Recovered fat yields and fatty acid profiles of White Teatfish and Elephant Trunkfish obtained from three extraction solvents.

\begin{tabular}{lccc}
\hline Type of fatty acid & MtOH:Chlo & EtOH:Chlo & EtOH:Hex \\
\hline White Teatfish & & & \\
Yield (wt\% dried & & & \\
weight) & $0.49 \pm 0.02$ & $0.50 \pm 0.01$ & $0.78 \pm 0.11$ \\
$\Sigma$ SFA & $49.41 \pm 1.03$ & $46.97 \pm 4.74$ & $24.58 \pm 0.65$ \\
$\Sigma$ USFA & $45.39 \pm 1.17$ & $43.76 \pm 3.23$ & $74.54 \pm 0.76$ \\
$\quad \Sigma(\omega-3)$ & $4.67 \pm 0.48$ & $4.62 \pm 0.73$ & $11.13 \pm 0.17$ \\
$\quad \Sigma(\omega-6)$ & $15.97 \pm 0.75$ & $18.97 \pm 4.6$ & $37.19 \pm 0.73$ \\
$\quad \Sigma(\omega-9)$ & $3.3 \pm 1.19$ & $7.82 \pm 4.1$ & $21.29 \pm 0.96$ \\
\hline Elephant Trunkfish & & & \\
Yield $(w t \%$ dried & $0.59 \pm 0.13$ & $0.58 \pm 0.10$ & $0.56 \pm 0.11$ \\
weight $)$ & $39.28 \pm 4.08$ & $36.38 \pm 4.47$ & $39.72 \pm 1.52$ \\
$\Sigma$ SFA & $56.56 \pm 2.15$ & $58.19 \pm 2.77$ & $55.61 \pm 2.74$ \\
$\Sigma U S F A$ & $7.03 \pm 0.57$ & $8.18 \pm 0.57$ & $5.56 \pm 0.91$ \\
$\quad \Sigma(\omega-3)$ & $24.7 \pm 4.63$ & $24.3 \pm 4.58$ & $24.77 \pm 1.45$ \\
$\quad \Sigma(\omega-6)$ & $10.6 \pm 1.81$ & $10.34 \pm 3.36$ & $13.9 \pm 4.33$ \\
\hline$(\omega-9)$ & & &
\end{tabular}

In Elephant Trunkfish, all three mixed solvents provided almost the same amounts of SFAs (36.38 to $39.72 \mathrm{wt} \%$ ) and USFA (55.56 to $58.19 \mathrm{wt} \%$ ) (Table 3). Against the result of the White Teatfish, the solvent EtOH:Hex provided the lowest amount of $\omega^{-3}$ fatty acids with $5.56 \mathrm{wt} \%$. The same pattern was observed in $\omega-6$ (ranging from $24.30 \mathrm{wt} \%$ to $24.77 \mathrm{wt} \%$ ) (Table 3). These results suggested that the selection of suitable solvent could be different depending on the types of sea cucumbers in terms of yield and fatty acid profiles.

\subsection{Analysis of sea cucumber collagen}

Since sea cucumbers have high collagen contents, thus it could be potential raw materials for collagen production. However, before using sea cucumber's collagens commercially, the property analysis should be conducted because they have wide variations and biological functions.

In this work, collagens of 4 different types of sea cucumbers, including Blackfish, Elephant Trunkfish, Black Teatfish and White Teatfish, were extracted by enzymatic and chemical extraction methods. Using SDSPAGE analysis, the molecular sizes of extracted collagens could be determined by comparing with the sizes of standard protein markers. The major protein bands with $\sim 48 \mathrm{kDa}$ - and $\sim 63 \mathrm{kDa}$-sized were found and in the chemical extraction. Whereas, $\sim 120 \mathrm{kDa}$-sized band was observed in the enzymatic extract. These results suggested 
that the enzymatic method was the better method to extract intact collagen due to the size of the collagen in SDS-PAGE [17, 22]. The hydrolyzed or degraded forms of collagens were observed in chemical extracts.

The chemical and physical properties of collagen extracts obtained from sea cucumbers were analyzed and compared by using FT-IR (Fig. 2). All chemical and all enzymatic extracts showed the typical peaks at about 3400 $\mathrm{cm}^{-1}$, which belonged to stretching vibration of $-\mathrm{N}-\mathrm{H}$ bonds, and the peak at $2900 \mathrm{~cm}^{-1}$, which represented to stretching vibration of $-\mathrm{C}-\mathrm{H}$ bonds [17]. Many peaks were found in the range starting from about $1000 \mathrm{~cm}^{-1}$ to $1600 \mathrm{~cm}^{-1}$, which belonged to the vibration of stretching and bending of amide $\mathrm{III}-\mathrm{C}=\mathrm{O}$ bonds, stretching of amide III $-\mathrm{N}-\mathrm{H}$ bonds, bending of $-\mathrm{C}-\mathrm{H}$ bonds and stretching of amide III $-\mathrm{C}-\mathrm{N}$ bonds $[17,23]$. These peaks indicate a high level of glycine and proline residues enriched in the collagen [17]. The last peak could be found at about 600 $\mathrm{cm}^{-1}$, which indicated the bending vibration of amide IV C-N bonds (Fig. 2). A comparison of these results with the standard curve from collagen showed that they have the same peak pattern and, therefore, all samples contained collagen.

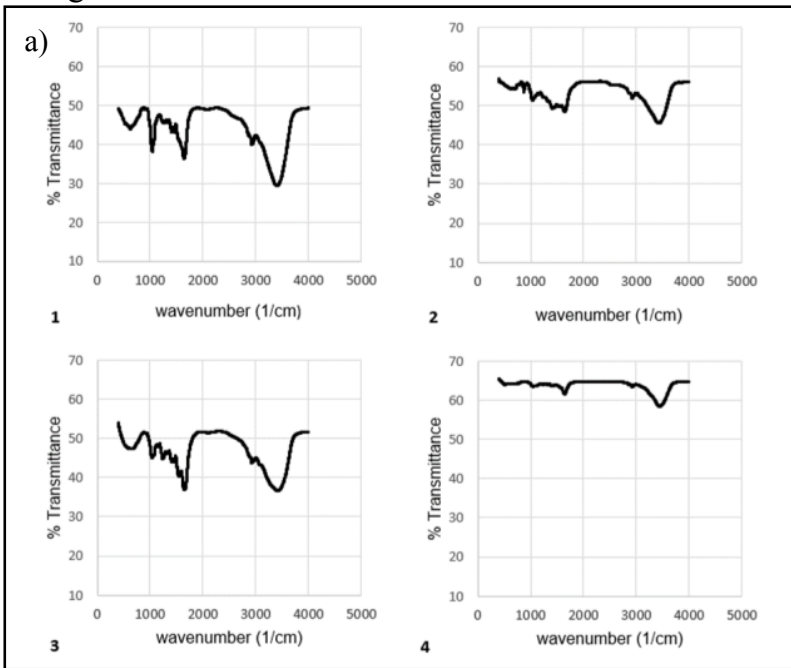

b)

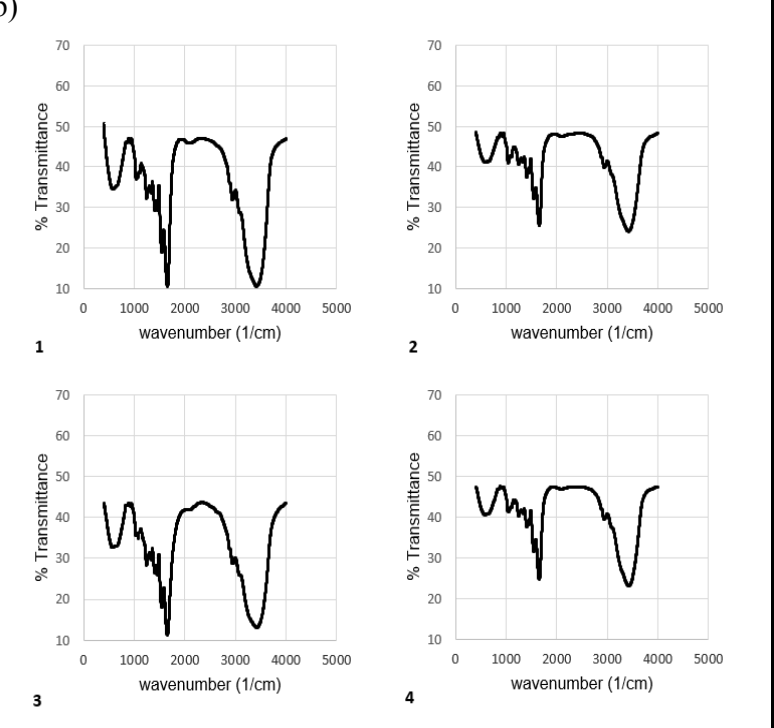

Fig. 2. FT-IR analysis a) chemical extraction b) enzymatic extraction (1=Blackfish, 2=Elephant Trunkfish, 3=Black Teatfish, $4=$ White Teatfish).
Nevertheless, the enzymatic collagen extraction delivered a better and consistent result of collagen extraction, because all the curves of the enzymatic extraction had the same peak patterns (Fig. 2). In chemical extraction, the height of each peak pattern varied depending on each type of sea cucumber. The Blackfish and the Black Teatfish had a similar shape with bigger peaks than the ones from Elephant Trunkfish and White Teatfish. In case of the White Teatfish the peaks in the area between $1000 \mathrm{~cm}^{-1}$ to $1500 \mathrm{~cm}^{-1}$ were so small that they cannot be clearly seen (Fig. 2).

\section{Conclusion}

In this study, the fatty acids and collagens of 4 different types of sea cucumbers were analyzed in order to investigate their potential use as materials for functional foods and ingredients. Comparison of fat yields, fatty acid profiles, simplicity and time-efficiency suggested that the suitable extraction method was Bligh and Dyer. Three types of mixed solvent systems for fat extraction shown to be efficient ones for fat extractions were $\mathrm{MtOH}$ :Chlo, EtOH:Chlo and EtOH:Hex due to high proportion of essential fatty acids, especially $\omega-3$ in the products. For collagen extraction, both FT-IR and SDS-PAGE agreed well to that enzymatic extraction method showed the better result with intact collagen compared to chemical extraction method. The analysis results in this work, which identified the beneficial nutrition of sea cucumbers, could be used as an initial investigation to evaluate the potential of sea cucumbers in food, cosmetic and pharmaceutical industries.

Authors would like to thank to Wonnapob Co., Ltd., Thailand Science Research and Innovation (TSRI, Research grant number RDG6250062) and King Mongkut's University of Technology North Bangkok (Research grant number KMUTNB-62-KNOW01 ) for financial support for this work.

\section{References}

1. A. Mazzocchi, C. Venter, K. Maslin, C. Agostoni, Nutrients 9, 8 (2017)

2. R. Ray, D. Newbold, D. Friesen, S. McCray, L. Ray, Bend Research Inc. (2000)

3. G. Blunden, Phytother Res. 15 (2001)

4. S. Bordbar, F. Anwar, N. Saari, M. Drugs 9, 10 (2011)

5. S.Y. Park, H.K. Lim, S. Lee, H.C. Hwang, S.K. Cho, M. Cho, Food Chem. 132 (2012)

6. X.Q. Zhou, C.H. Wang, A.L. Jiang, Adv Mater Res. 393 (2012)

7. X.Q. Zhou, C.H. Wan, A.L. Jiang, Eur Food Res Technol. 234 (2012)

8. B. Forghani, A. Ebrahimpour, J. Bakar, A.A. Hamid, Z. Hassan, N. Saari, Altern Med. 9 (2012)

9. D. Swanson, R. Block, A. Shaker, Mousa. Adv Nutr 3, 1 (2012) 
10. R. Pangestuti, Z. Arifin, J. Tradit. Complement Med 8, 3 (2018)

11. W. Vergara, A. Rodríguez, Nat Resour. 7 (2016)

12. A. Ceesay, M.N. Shamsudin, M. Aliyu-Paiko, I.S. Ismail, M.F. Nazarudin, N.M. Alipiah, BioMed Res. Int. 2019, 1640684 (2019)

13. E. G. Bligh, W.J. Dyer, Can J Biochem Physiol. 37 (1959)

14. K.H. Dewi, M. Markom, W.R. Wan Daud, Adv Mat Res. 9, (2011)

15. P. Rachamontree, M. Sriariyanun, S. Tepaamorndech, I. Somboonwatthanakul, Orient J Chem. 35, 2 (2019)

16. S. Chaichoowong, J.B. Bol, P. Bol, T. Gamse, M. Sriariyanun, Orient J Chem. 33, 1 (2017)
17. F. Liu, L. Zamora, A. Jeffs, S.Y. Quek, Nutrire 42, 12 (2017)

18. L. Switzar, M. Giera, W. Niessen, J Proteome Res. 12 (2013)

19. M. Sriariyanun, P. Tantayotai, P. Yasurin, P. Pornwongthong, K. Cheenkachorn, Electron J Biotechnol. 19, 1 (2016)

20. A.P. Simopoulos, Nutrients 2016, (2016)

21. D. Perrin, Springer (1981)

22. F. Lui, L. Zamora, A. Jeffs, S.Y. Quek, Nutrire (2017)

23. M. Zhong, T. Chen, C. Hu, C. Ren, J Food Sci. 80, 4 (2015) 